\title{
Marnix Dressen, Jean-Pierre Durand (coord.), La violence au travail
}

Toulouse, Octarès éditions, collection « Le travail en débats ", série «Colloques et congrès », 2011

\section{Catherine Cavalin}

\section{CpenEdition}

\section{Journals}

Édition électronique

URL : http://journals.openedition.org/travailemploi/5449

DOI : 10.4000/travailemploi.5449

ISSN : 1775-416X

Éditeur

DARES - Ministère du Travail

\section{Édition imprimée}

Date de publication : 15 novembre 2011

Pagination : 86-87

ISBN : 0224-4365

ISSN : 0224-4365

\section{Référence électronique}

Catherine Cavalin, « Marnix Dressen, Jean-Pierre Durand (coord.), La violence au travail », Travail et Emploi [En ligne], 128 | octobre-décembre 2011, mis en ligne le 01 juillet 2012, consulté le 22 septembre 2020. URL : http://journals.openedition.org/travailemploi/5449 ; DOI : https://doi.org/ 10.4000/travailemploi.5449

Ce document a été généré automatiquement le 22 septembre 2020

(c) Direction de l'animation de la recherche, des études et des statistiques (Dares) 


\section{Marnix Dressen, Jean-Pierre Durand (coord.), La violence au travail}

Toulouse, Octarès éditions, collection « Le travail en débats », série «Colloques et congrès », 2011

\section{Catherine Cavalin}

\section{RÉFÉRENCE}

Marnix Dressen, Jean-Pierre Durand (coord.), La violence au travail, Toulouse, Octarès éditions, collection « Le travail en débats », série « Colloques et congrès », 2011

1 Issu des travaux du réseau thématique «Travail, organisations, emploi » (le « RT25 ») de l'Association française de sociologie, cet ouvrage collectif coordonné par Marnix DRESSEN et Jean-Pierre DURAND fait suite au $3^{e}$ congrès de l'AFS («Violences et société ») qui s'est tenu en 2009. Le volume se propose d'embrasser la violence au travail dans une grande diversité de formes. Le titre de la première («Les formes renouvelées de la violence au travail ») des cinq parties que compte l'ouvrage informe, à bien des égards, sur l'ensemble du contenu du volume, sans que l'objectif visé soit celui d'un inventaire exhaustif des types de violences observés en lien avec l'activité de travail. Formes diverses de la violence au travail et nouveautés de celles-ci : voilà ce qu'ont pour mission de questionner les nombreux auteurs qui se partagent vingt-huit chapitres.

Le lecteur attend par conséquent d'apprendre ce qu'il y a de violent dans le travail et ce que cette violence a de nouveau; autrement dit, de comprendre dans quel temps long la question de la violence au travail peut être saisie. À cette question de l'historicisation $\mathrm{du}$ problème traité, les auteurs répondent chemin faisant plutôt que de manière systématique. La lecture permet d'accumuler des traits qui finissent par composer le tableau du moment historique présent de la violence au travail. Nous comprenons ainsi que nous nous situons au confluent des changements dans l'usage des techniques au travail (diffusion et nouveaux usages des technologies de l'information et de la communication particulièrement), des changements dans l'organisation du travail, 
dans les modes de gestion qui le gouvernent (nouveau ou « néo-» management), le tout à un moment où le lieu commun indiscutable est, pour tous les auteurs, celui de l'intensification $\mathrm{du}$ travail constatée depuis le milieu des années $1990^{1}$. Bouleversements organisationnels, techniques et managériaux font l'objet de la plus grande attention, comme autant de sources de tensions qui rendent possibles la reviviscence et les transformations de ce que le travail peut avoir ou susciter de violent pour ceux qui y sont engagés. Car, nous le comprenons également de-ci de-là, il n'est pas question de dire que la violence serait, en elle-même, une nouveauté pour le travail : le travail est lui-même une violence (p. 98), et un retour répété à la notion marxienne d'aliénation (p. 24 et 366 par ex.) permet notamment de comprendre certaines manifestations historiques de la violence faite aux travailleurs dans les rapports de production capitalistes. Saisir la diversité des formes de violence au travail à la fin des années 2000 en France, c'est se situer dans le prolongement de ces analyses, à un moment que les auteurs jugent pertinent pour mettre en lumière comment la domination contenue historiquement dans le rapport salarial passe aujourd'hui par des voies différentes, moins manifestes. L'analyse porte ainsi sur «des épreuves comparables à celles qui ont touché les ouvriers de l'industrie quarante ans plus tôt » (p. 218), mais dans « un nouveau régime de subordination» (p. 77). Les terrains sont par exemple ceux de la mobilité professionnelle ou géographique contrainte (pp.115-125 par ex.), des procédures d'évaluation individualisée (pp.199-210), de la sélection à l'embauche (pp.147-156) ou de la pratique du sport en entreprise (pp. 73-85).

3 À la recherche d'un élément qui suffirait presque à décrire ce que les formes de violence au travail ont de nouveau et de spécifique, le lecteur peut s'arrêter à la notion, qui traverse presque tous les chapitres, de violence "symbolique». Un comptage lexicométrique des nombreux adjectifs qualificatifs qui accompagnent le substantif «violence(s) » dans l'intégralité du volume donnerait très certainement la première place à celui-ci. La plupart des textes se réfèrent explicitement à l'acception bourdieusienne de la notion qui fait ici consensus, maintes fois citée dans sa définition littérale, et mobilisée y compris comme point d'orgue conclusif de l'un ou l'autre chapitre (p. 124 ou 251 plus encore). De là découlent plusieurs objets d'analyse : «le régime contemporain de mobilisation de la force de travail [jouant] de la violence symbolique avec une intensité inédite » (p. 59), la violence au travail doit être étudiée dans sa douceur et son invisibilité, sa discrétion si différente de l'éclatante survenue de la violence physique ou verbale, sa capacité à pervertir les victimes en vecteurs mêmes (sur eux et sur les autres, même dans des relations de travail horizontales) de la violence qu'elles subissent (p. 139 par ex.). Un mécanisme essentiel est donc aussi celui du consentement, désigné en tant que tel (p.123) ou à travers des références à la littérature (Michael BURAWOY pour le texte de Jean-Pierre DURAND). Lorsque, pour de rares auteurs, la violence symbolique n'est pas l'angle d'attaque privilégié ou exclusif, ils s'en expliquent (p. 128 ou 297). Jean-Pierre DURAND est de ceux-là, qui propose d'utiliser les notions de violence «normative » ou « de normation » (en rapport direct avec le «manufacturing consent» de Burawoy, justement) pour mieux penser le processus d'évaluation des salariés.

4 Les textes qui composent l'ouvrage renvoient aussi fréquemment aux ouvrages bien connus de Luc BOLTANSKI et Laurent THÉVENOT d'une part, Luc BOLTANSKI et Ève CHIAPELLO d'autre part ${ }^{2}$, même si ces deux renvois sont plus discrets que la référence presque 
omniprésente à Pierre BOURDIEU et moins évidemment centraux dans la réflexion. Une seule contribution (pp. 157-167) se saisit plus frontalement de ce que cette « sociologie pragmatique » peut apporter à la compréhension des situations de violence au travail : quelles sont les épreuves qui se jouent, quels en sont les protagonistes et dans quelles conditions peuvent-ils y participer? Pour répondre, Damien CARTRON et Michel GOLLAC soulignent les capacités critiques des acteurs et les rapportent à des conditions de travail: la routine industrielle pour les «équipiers" McDo, les situations plus dramatiques liées aux accidents de travail, la délimitation des épreuves qui intervient dans les interactions entre hommes et machines. Ici, l'analyse pragmatique peut aider à repérer et comprendre une violence à la fois « ouverte » et « symbolique » au travail.

5 Le catalogue des points d'appui que trouve cet ouvrage dans la littérature pourrait s'attarder ailleurs, en particulier sur deux autres veines d'analyse. Le premier des deux ponts est lancé vers Christophe DEJours (surtout), Yves CLOT et la psychodynamique du travail. Les emprunts, parfois directs (p. 241) et relativement nombreux, surtout dans le dernier tiers de l'ouvrage, sont toutefois prudents. Le refus d'une analyse qui passerait par la psychologisation des rapports sociaux est en effet réitéré, Marnix DRESSEN retenant même ce credo en conclusion, pour en marquer sans doute l'importance. Est ici revendiquée une "lecture organisationnelle et politique du mal-être au travail» (p. 182). En définitive, on peut se demander si la «violence» et la "souffrance» peuvent être mobilisées avec profit par la sociologie du travail, les auteurs d'une part nous livrant leur difficulté à tenir fermement l'objet toujours fuyant qu'est la notion de violence (pp. 110-111 ou p. 312), les liens entre violence et souffrance restant d'autre part à affermir (p.315). Un second pont est tendu vers l'analyse des risques psychosociaux «à la KARASEK-SIEGRIST»: demande psychologique, latitude professionnelle et soutien social sont là, en filigrane ou désignés autrement (rôle du « collectif de travail » par exemple), parfois encore explicitement utilisés (pp. 223-234). On aimerait en savoir plus, en particulier parce que la mobilisation du modèle de KARASEK va parfois de pair avec l'idée, présentée presque comme un postulat, des liens (pourtant si problématiques à démontrer) entre violences subies et état de santé.

Ces deux derniers points conduisent à suggérer des pistes qu'un dialogue entre sociologie du travail, de la violence et de la santé pourrait utilement développer. Tout d'abord, la plupart des contributions reposent sur des recherches de nature qualitative. Les enquêtes quantitatives sont parfois convoquées (pp. 39-51), mais elles demeurent plutôt de lointaines sources d'illustration ou de cadrage du propos. Or, plusieurs enquêtes statistiques sur les violences ont été menées depuis l'Enquête nationale sur les violences envers les femmes en France (ENVEFF) ${ }^{3}$, qui toutes se sont confrontées à la question de la définition et de la mesure des violences. Un échange avec ces travaux serait riche pour tous, autorisant notamment à approfondir la dialectique entre objectivité et subjectivité de la violence subie. Dialoguer avec les analyses quantitatives récentes permettrait également à la fois de mieux cerner la complexité des relations avec la santé, dans une approche plus critique des catégories de "stress", « dépression »... et, sans tomber dans la psychologisation redoutée, d'ouvrir à d'autres perspectives qu'organisationnelles, techniques ou gestionnaires. Quid, par exemple, des rapports de genre dans l'explication de la violence au travail ? On attend, enfin, que la découverte de la diversité des formes de violence au travail pointe vers les horizons plus ouverts que suggèrent quelques auteurs: dans quelle mesure les «maux du travail » sont-ils des catégories construites? Peut-on s'autoriser, sans autre forme de 
procès, à les considérer comme le résultat évident de l'intensification du travail et de ses avatars ${ }^{4}$ ? Peut-on, autrement dit, traiter du "malaise dans la société » (précarité, vulnérabilité, souffrance, violence, pénibilité...) sans craindre de lâcher la proie pour l'ombre? Alain EHRENBERG, lui aussi souvent cité dans cet ouvrage, nous invite à faire de ce malaise "moins un point de départ de l'analyse sociologique dont le sociologue aurait à explorer les causes, qu'un problème à élaborer et à clarifier $»^{5}$.

\section{NOTES}

1. GollaC M., volKoff S. (1996), "Citius, altius, fortius. L'intensification du travail», Actes de la recherche en sciences sociales, $\mathrm{n}^{\circ} 114$, septembre, pp. 54-67.

2. BOLTANSKI L., THÉVENOT L. (1991), De la justification. Les économies de la grandeur, Paris, Gallimard (NRF Essais), 496 p., et BOLTANSKI L., CHIAPELLO E. (1999), Le nouvel esprit du capitalisme, Paris, Gallimard (NRF Essais), $848 \mathrm{p}$.

3. JASPARD M. et al, (2003), Les violences envers les femmes en France. Une enquête nationale, Paris, la Documentation française, $370 \mathrm{p}$.

4. LALLEMENT M., MARRY C., LORIOL M., MOLINIER P., GOLLAC M., MARICHALAR P., MARTIN E., (2011), « Maux du travail : dégradation, recomposition ou illusion? », Sociologie du travail, vol. 53, n 1, pp. 3-36.

5. EHRENBERG A. (2011), «La société du malaise. Une présentation pour un dialogue entre clinique et sociologie ", Adolescence, vol. 29, n 3, pp. 553-570.

\section{AUTEURS}

\section{CATHERINE CAVALIN}

Centre d'études de l'emploi 